\title{
CREEP FAILURE TIME OF THIN-WALLED PIPES UNDER COMBINED INTERNAL PRESSURE, BENDING AND TENSION
}

\author{
A. EI Megharbel \\ Suez Canal University, Faculty of Engineering, Production Engineering and Mechanical \\ Design Department, Port Said 42523, Egypt
}

\begin{abstract}
The creep failure time is calculated for rectilinear thin-walled pipes subjected to combined internal pressure and both of tension and bending. The calculating of the failure time is given using the concept of equivalents stresses. The equivalent stresses are found from the mixed delayed-failure criterion relating the maximum normal stress and the intensity of tangent stresses. The obtained results were compared with the results obtained by Golub et al. (2004) [2] for pipes under only couple stresses. The present work can be used to calculate the time to creep failure of thin-walled pipes under internal pressure in combination with axial load and binding.

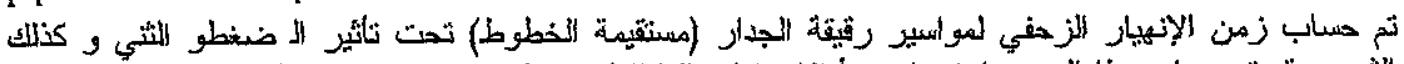

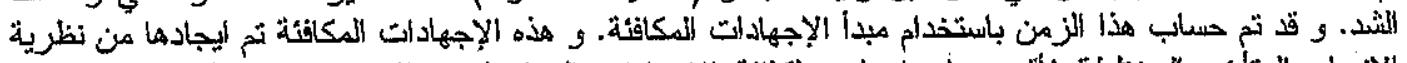

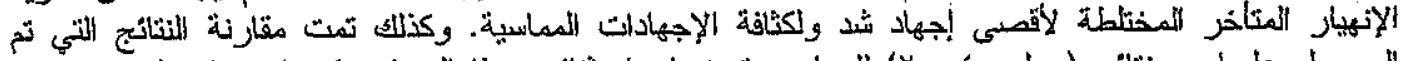

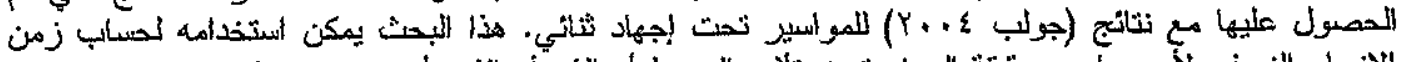

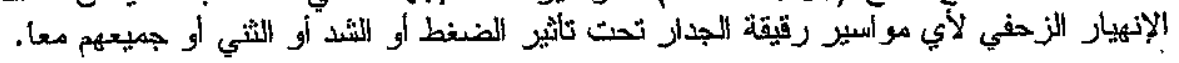

Keywords: Creep failure, internal pressure, plane bending, thin-walled pipe, uniaxial tension

\section{INTRODUCTION}

Elements of steam and gas turbines, jet engines, steam boilers, rockets, oil and gas processing plants are widely used of thin-walled pipes. These elements could subject to creep; therefore the creep analysis is a great interested field [1]. The time to failure $t_{R T}$ of a thin-walled pipe under internal pressure $(p)$ and both of axial force $(N)$ and bending moment $\left(M_{B}\right)$ is the objective of this paper.

In [2], [3] and [4], a mixed plane-stress criterion of delayed failure was established and experimentally validated for metal and polymeric materials. The criterion has the form of two-parameter linear interpolation relating two stress invariants that represent ductile and brittle fracture and accounting for the signs of the principal stresses. However, Golub et al. used the concept of unified limit stress diagram to model creep-fatigue interaction [5]. In addition, Experimental analysis of high-temperature creep, fatigue and damage has been done by [6]. A technique of constructing unified deformation and damage diagrams based on the conditions of proportional similarity is substantiated.

Creep deformation and damage of High Speed Steel HS 6-5-3 was investigated by numerical simulation by Hofter et al. [7]. Whereas, Cole and Bhadeshia [8] tried to estimate of the creep rupture strength of heat resistant steels and welding. Cole deals with quantitative methods for the design of steel weld metals for elevated temperature applications.
A series of fatigue tests under uniaxial and torsional loading at constant room temperature is carried out by [9]. A cyclic constitutive and damage model is presented to describe the characterization of stress strain response and damage evolution for these fully reversed strain controlled tests.

Tinga et al. [10] are proposed a damage model for single crystal Ni-base superalloys that integrates time-dependent and cyclic damage into a generally applicable time-incremental damage rule. Yang et al. [11] are proposed a simple stress controlled fatiguecreep damage evolution model, based on the ductility dissipation theory and effective stress concept of continuum damage mechanism and damage constants can be obtained through fatigue-creep tests directly.

An advanced elasto-viscoplastic model for the timevarying response of Ultra-high molecular weight polyethylene (UHMWPE) was used by Bischoff [12] to explore the effects of loading frequency and creep time on the material behavior during cyclic loading. Whereas, an integral computational method has been developed to provide initial values to a subsequent fitting of creep data based on non-linear and iterative methods by Rieiro et al. [13]. Kachanoy [14] has suggested a theoretical model for the time to rupture with the account of embrittlement.

A method for the estimation of the time to failure under creep conditions is proposed by Zharkova and Botvina [15]; it is based on the approach of a phase 
transition theory and the similarity of fracture mechanisms.

The equivalent-stress methods of long-term strength analysis of pipes are the most efficient and widely used in current design practice. The accuracy of calculations strongly depends on the adequacy of the equivalent stresses to the stress and failure modes of the pipe and on the degree of agreement between the material constants and long-term strength characteristics obtained under uniaxial tension

\section{ANALYSIS}

This paper is interested in thin-walled pipe under internal pressure and axial load as well as under bending.

2.1 The Failure of Thin-Walled Pipes under Internal Pressure (Golub et al. analysis [2])

\subsection{Failure under internal pressure}

Golub et al. [2] have derived a formula to evaluate the creep time for pipes under internal pressure only.

$$
\begin{aligned}
& \sigma_{1}=\frac{p D_{m}}{2 h}, \quad \sigma_{2}=\frac{p D_{m}}{4 h}, \quad \sigma_{3}=0 \\
& t_{R T}=\frac{1}{B[(1+\alpha) \lambda]^{m}\left(\frac{p}{2}\right)^{m}}
\end{aligned}
$$

Where $\lambda=\frac{D_{m}}{2 h}$

The material constant $\alpha$ is

$$
\alpha=\frac{2 \sigma_{1}-\lambda p_{1}}{\sigma_{t}}
$$

\subsubsection{Failure under internal pressure and axial load}

For a rectilinear thin-walled pipe under internal pressure $p$ and axial tensile force $N$, the equilibrium conditions for the pipe yield

Case a $\delta \leq \frac{\lambda}{2}$

$\sigma_{1}=\frac{p D_{m}}{2 h}, \quad \sigma_{2}=\frac{p D_{m}}{4 h}+\frac{N}{\pi D_{m} h}, \quad \sigma_{3}=0$

For $\frac{N}{\pi D_{m} h} \leq \frac{p D_{m}}{4 h}$

The creep time is given as

$t_{R T}=\frac{1}{B}\left[\frac{\sqrt{3 \lambda^{2}+4 \delta^{2}}+\left(2 \sqrt{3} \lambda-\sqrt{3 \lambda^{2}+4 \delta^{2}}\right) \alpha}{\sqrt{3}}\right]^{-m}\left(\frac{p}{2}\right)^{-m}$

Where $\frac{N}{\pi D_{m} h p}=\delta$ $\alpha=\frac{\lambda\left(2 \sqrt{3} \sigma_{t}-\sqrt{3 \lambda^{2}+4 \delta^{2}} p_{t}\right)}{\left(2 \sqrt{3} \lambda-\sqrt{3 \lambda^{2}+4 \delta^{2}}\right) \sigma_{t}}$

Case b $\delta \geq \frac{\lambda}{2}$

The equilibrium conditions for the pipe yield

$\sigma_{1}=\frac{p D_{m}}{4 h}+\frac{N}{\pi D_{m} h}, \quad \sigma_{2}=\frac{p D_{m}}{2 h}, \quad \sigma_{3}=0$

For $\frac{N}{\pi D_{m} h} \geq \frac{p D_{m}}{4 h}$

The formula for creep time is:

$t_{R T}=\frac{1}{B}\left[\frac{\sqrt{3 \lambda^{2}+4 \delta^{2}}+\left(\sqrt{3} \lambda+2 \sqrt{3} \delta-\sqrt{3 \lambda^{2}+4 \delta^{2}}\right) \alpha}{\sqrt{3}}\right]^{-m}\left(\frac{p}{2}\right)^{-1 m}$

and

$\alpha=\frac{(\lambda+2 \delta)\left(2 \sqrt{3} \sigma_{t}-\sqrt{3 \lambda^{2}+4 \delta^{2}} p_{t}\right)}{2\left[\sqrt{3}(\lambda+2 \delta)-\sqrt{3 \lambda^{2}+4 \delta^{2}}\right] \sigma_{t}}$

2.1.3 Failure under internal pressure and Bending For a rectilinear thin-walled pipe under internal pressure $p$ and bending moment $\mathrm{M}_{\mathrm{B}}$, the equilibrium conditions for the pipe yield

Case a $\xi \leq \frac{\lambda}{2}$

$\sigma_{1}=\frac{p D_{m}}{2 h}, \quad \sigma_{2}=\frac{p D_{m}}{4 h}+\frac{2 M_{B}}{\pi D_{m}^{2} h}, \quad \sigma_{3}=0$

For $\frac{2 M_{B}}{\pi D_{m}^{2} h} \leq \frac{p D_{m}}{4 h}$

And the creep time is given as

$t_{R T}=\frac{1}{B}\left[\frac{\sqrt{3 \lambda^{2}+4 \xi^{2}}+\left(2 \sqrt{3} \lambda-\sqrt{3 \lambda^{2}+4 \xi^{2}}\right) x}{\sqrt{3}}\right]^{-m}\left(\frac{p}{2}\right)^{-m}$

$\alpha=\frac{\lambda\left(2 \sqrt{3} \sigma_{1}-\sqrt{3 \lambda^{2}+4 \xi^{2}} p_{t}\right)}{\left(2 \sqrt{3} \lambda-\sqrt{3 \lambda^{2}+4 \xi^{2}}\right)}$

Where $\frac{2 M_{B}}{\pi D_{m}^{2} h p}=\xi$

Case b $\xi \geq \frac{\lambda}{2}$

The equilibrium condition

$\sigma_{1}=\frac{p D_{m}}{4 h}+\frac{2 M_{B}}{\pi D_{m}^{2} h}, \quad \sigma_{2}=\frac{p D_{m}}{2 h}, \quad \sigma_{3}=0$

For $\frac{2 M_{B}}{\pi D_{m}^{2} h} \geq \frac{p D_{m}}{4 h}$

The creep time is 
$t_{R T}=\frac{1}{B}\left[\frac{\sqrt{3 \lambda^{2}+4 \xi^{2}}+\left(\sqrt{3} \lambda+2 \sqrt{3} \xi-\sqrt{3 \lambda^{2}+4 \xi^{2}}\right) \alpha}{\sqrt{3}}\right]^{-m}\left(\frac{p}{2}\right)^{-m}$.

$$
\alpha=\frac{(\lambda+2 \xi)\left(2 \sqrt{3} \sigma_{t}-\sqrt{3 \lambda^{2}+4 \xi^{2}} p_{t}\right)}{2\left[\sqrt{3}(\lambda+2 \xi)-\sqrt{3 \lambda^{2}+4 \xi^{2}}\right] \sigma_{r}} .
$$

\subsection{Formulation of the Problem and the Initial Relations (The Present Work)}

Consider a long rectilinear thin-walled pipe of circular cross section under creep conditions. Denote the diameter of the median surface by $\mathrm{D}_{\mathrm{m}}$ and wall thickness, which is constant, by $h$. It is assumed that $2 \mathrm{~h}<<\mathrm{D}_{\mathrm{m}}$.

The pipe is subjected to internal pressure $p$ in combination with axial tensile force $N$ and bending moment $\mathrm{M}_{\mathrm{B}}$. Under creep, the external load remains constant. The ends of the pipe are not restrained, and its deformation is free. The material of the pipe is homogeneous, isotropic, and incompressible, and its initial state is elastic. The time to failure $t_{R T}$ of the pipe is found using the approach Golub et al. (2004) based on the concept of equivalent stress as some scalar characteristic of the initial stress of the pipe. The equivalent stress relates the failure of the pipe under arbitrary stress and the failure of a cylindrical specimen under uniaxial tension. Therefore

$$
t_{R}=\frac{1}{B\left(\sigma_{t}\right)^{m}} \Rightarrow t_{R T}=\frac{1}{B\left(\sigma_{e q v}\right)^{m}}
$$

Where $t_{R}$ and $\sigma_{t}$ are the time to failure and failure stress of smooth cylindrical specimens under uniaxial tension; $\sigma_{\mathrm{eq} y}$ is the equivalent stress; $\mathrm{B}$ and $\mathrm{m}$ are material constants determined from standard uniaxial-tension creep-rupture tests on smooth cylindrical specimens. In what follows, we consider that for the values of $B$ and $m$. found, the delayedfailure patterns of smooth specimens and thin-walled pipes are identical. If the standard long-term strength curve has breaks, then the values of $B$ and $m$ are calculated for each section of the curve.

The combination of internal pressure, tension, and bending induces plane stress in thin-walled pipes. A mixed delayed-failure criterion in the form Golub et al. (2003).

$\sigma_{e q p}= \begin{cases}\alpha \sigma_{\max }+(1-\alpha) s_{1} & \text { for } \sigma_{1}>\sigma_{2}>0, \sigma_{3}=0 \\ 2 \beta \tau_{\max }+(1-\beta) \tau_{o c t} & \text { for } \sigma_{1}>0, \sigma_{2}=0, \sigma_{3}<0\end{cases}$

can be used as equivalent stress $\sigma_{\mathrm{eqv}}$. This criterion accounts for the signs of the principal stresses and relates the maximum normal stress $\sigma_{\max }$,

$\sigma_{\max }=\sigma_{1}$ $s_{i}=\frac{1}{\sqrt{3}} \sqrt{\sigma_{1}^{2}-\sigma_{1} \sigma_{2}+\sigma_{2}^{2}}$

the double maximum tangential stress $2 \tau_{\max }$,

$2 \tau_{\max }=\sigma_{1}-\sigma_{2}$

and the octahedral tangential stress $\tau_{\text {oct }}$,

$\tau_{o c t}=\frac{\sqrt{2}}{3} \sqrt{\sigma_{1}^{2}-\sigma_{1} \sigma_{2}+\sigma_{2}^{2}}$

where $\sigma_{1}$ and $\sigma_{2}$ are the principal normal stresses $\left(\sigma_{1}\right.$ $>\sigma_{2}$ ); and $\alpha$ and $\beta$ are experimentally determined material constants reflecting the effect of the mode of plane stress $\left(\sigma_{2}\right.$ is any nonzero second principal stress). When thin-walled pipes are subjected to internal pressure in combination with both tension and bending, the signs of the principal stresses coincide. Substituting the first relation in (20) into Eq. (19) and taking (21) and (22) into account, we obtain an equation for the time to failure in terms of principal stresses

$$
t_{R T}=\frac{1}{B\left[\frac{\sqrt{3} \alpha \sigma_{1}+(1-\alpha) \sqrt{\sigma_{1}^{2}-\sigma_{1} \sigma_{2}+\sigma_{2}^{2}}}{\sqrt{3}}\right]^{m}}
$$

which in fact determines the time of occurrence of local failure.

\subsubsection{Failure under internal pressure, tension and bending}

Consider a rectilinear thin-walled pipe with end plates under internal pressure $p$ and both axial load $N$ and bending moment $\mathrm{M}_{\mathrm{B}}$.We assume that the pipe is long. The plane stress state in the median surface of the pipe is membrane and statically determinate. The equilibrium conditions yield

$\sigma_{1}=\sigma_{\phi}=\frac{p D_{m}}{2 h}, \quad \sigma_{2}=\sigma_{z}^{*}=\frac{p D_{w t}}{4 h}+\frac{N}{\pi D_{m} h}+\frac{2 M_{R}}{\pi D_{m}^{2} h}, \quad \sigma_{3}=\sigma_{r}=0$

For $\frac{N}{\pi D_{m} h}+\frac{2 M_{B}}{\pi D_{m i}^{2} h} \leq \frac{p D_{m}}{4 h}$ and

Where $\sigma_{\varphi}$ is the hoop stress, is $\sigma_{z}$ the axial stress, and $\sigma_{\mathrm{r}}$ is the radial stress, while;

$\sigma_{1}=\frac{p D_{m}}{4 h}+\frac{N}{\pi D_{m} h}+\frac{2 M_{B}}{\pi D_{m}^{2} h}, \quad \sigma_{2}=\frac{p D_{m}}{2 h}, \quad \sigma_{3}=0$

For $\frac{N}{\pi D_{m} h}+\frac{2 M_{B}}{\pi D_{m}^{2} h} \geq \frac{p D_{m}}{4 h}$

Case a $\delta+\xi \leq \frac{\lambda}{2}$

Substituting Eq. (26) into Eq. (25) yields the time to failure $\delta+\zeta \leq \lambda / 2$

the intensity of tangential stresses, 


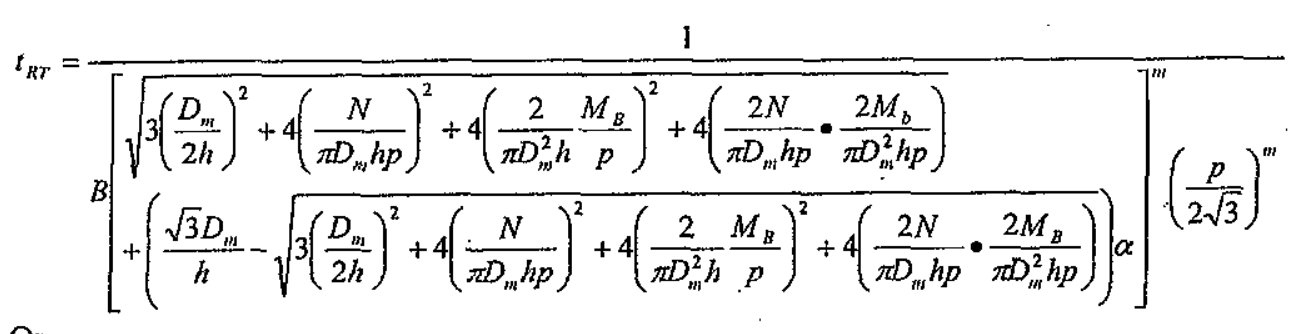

Or

$$
t_{R T}=\frac{1}{B}\left[\frac{\sqrt{3 \lambda^{2}+4 \delta^{2}+4 \xi^{2}+8 \delta \xi}+\left(2 \sqrt{3} \lambda-\sqrt{3 \lambda^{2}+4 \delta^{2}+4 \xi^{2}+8 \delta \xi}\right) \alpha}{\sqrt{3}}\right]^{-m}\left(\frac{p}{2}\right)^{-m}
$$

where $\lambda, \delta$ and $\xi$ are a dimensionless parameter, a measure of variation in the stress state of the thin-walled pipe under internal pressure and both axial load and bending (Eqs. (3), (7) and (15) respectively).

The material constant $\alpha$ in Eqs. (28) and (29) is determined from the relation in Golub et al. [3].

$$
\alpha=\frac{\lambda\left(2 \sqrt{3} \sigma_{t}-\left(\sqrt{3 \lambda^{2}+4 \delta^{2}+4 \xi^{2}+8 \delta \xi}\right) p_{t}\right)}{\left(2 \sqrt{3} \lambda-\sqrt{3 \lambda^{2}+4 \delta^{2}+4 \xi^{2}+8 \delta \xi}\right) \sigma_{i}}
$$

where $\sigma_{t}$ and $p_{t}$ are experimentally determined averaged (in view of the statistical properties of the material) long-term strengths, at the same time to failure, for a cylindrical specimen under uniaxial tension and a thinwalled pipe under internal pressure.

Case b $\delta+\xi \geq \frac{\lambda}{2}$

Substituting Eq. (27) into Eq. (25) yields the time to failure $\delta+\zeta \geq \lambda / 2$

$$
t_{R T}=\frac{1}{\left[\begin{array}{l}
\sqrt{\left(\frac{D_{m}}{2 h}\right)^{2}+4\left(\frac{N}{\pi D_{m} h p}\right)^{2}+4\left(\frac{2}{\pi D_{m}^{2} h} \frac{M_{B}}{p}\right)^{2}+8\left(\frac{2 M_{B}}{\pi D_{m}^{2} h p} \frac{N}{\pi D_{m} h p}\right)} \\
+\left(\begin{array}{l}
2 \sqrt{3}\left(\frac{D_{m}}{4 h}+\frac{N}{\pi D_{m} h p}+\frac{2 M_{B}}{\pi D_{m}^{2} h p}\right) \\
-\sqrt{\left(\frac{D_{m}}{2 h}\right)^{2}+4\left(\frac{N}{\pi D_{m} h p}\right)^{2}+4\left(\frac{2}{\pi D_{m}^{2} h} \frac{M_{B}}{p}\right)^{2}+8\left(\frac{2 M_{B}}{\pi D_{m}^{2} h p} \frac{N}{\pi D_{n} h p}\right)}
\end{array}\right]
\end{array}\right]^{m}}
$$

Or

$t_{R \gamma}=\frac{1}{B}\left[\frac{\sqrt{3 \lambda^{2}+4 \delta^{2}+4 \xi^{2}+8 \delta \xi}+\left(\sqrt{3}(\lambda+2 \delta+2 \xi)-\sqrt{3 \lambda^{2}+4 \delta^{2}+4 \xi^{2}+8 \delta \xi}\right) \alpha}{\sqrt{3}}\right]^{-m}\left(\frac{p}{2}\right)^{-m}$

The material constant $\alpha$ is determined from Golub et al. .

$$
\alpha=\frac{(\lambda+2 \delta+2 \xi)\left\{2 \sqrt{3} \sigma_{1}-\left(\sqrt{3 \lambda^{2}+4 \delta^{2}+4 \xi^{2}+8 \delta \xi}\right) p_{t}\right\}}{2\left[\sqrt{3}(\lambda+2 \delta+2 \xi)-2 \sqrt{3 \lambda^{2}+4 \delta^{2}+4 \xi^{2}+8 \delta \xi}\right]}
$$

where the notation is the same as in (30) and (32)

Substitute in Eq. (29) with $\delta=0$ and $\xi=0$ (i.e. pipe is under internal pressure only) yields to the same eq. (2). Otherwise substitute in Eq. (29) with $\delta=0$ yields the same Eq.(13), while substituting with $\xi=0$ yields Eq.(6). Also substitute in Eq. (30) with $\delta=0$ and $\xi=$ 0 yield also the same eq. (4). While substituting in Eq. (30) with $\xi=0$ yields to the same Eq.(8) and substituting with $\delta \approx 0$ yields the same Eq. (14) (i. e. the same work of Golub et al. [2]).
Substitute in Eq. (32) with $\delta=0$ yields the same Eq. (17), while substituting with $\xi=0$ yields Eq. (10). While substituting in Eq. (33) with $\xi=0$ yields to the same Eq. (11) and substituting with $\delta=0$ yields the same Eq. (18) (i. e. agreement with Golub et al. [2]). The critical state between case $a$ and $b$ is when $\delta+\xi=\frac{\lambda}{2}$, substitute with this value in Eqs. (29) and (32) yields the same following equation

$t_{R T}=\frac{1}{B}\left[\frac{4(\delta+\xi)(1+0.732 \alpha)}{\sqrt{3}}\right]^{-m}\left(\frac{p}{2}\right)^{-m}$

Which give a good impression to the calculation.

While substituting with $\delta+\xi=\frac{\lambda}{2}$ in Eqs. (30) and (33) yields the same following equation for $\alpha$ 


$$
\alpha=\frac{\left\{\sqrt{3} \sigma_{t}-2(\delta+\xi) p_{t}\right\}}{0.732 \sigma_{t}}
$$

This agreement is also good for the calculation.

\section{RESULTS AND DISCUSSION}

The time to creep failure of thin-walled pipes under internal pressure combined with both axial load and bending is introduced in the previous section. These analytical are concerned with long rectilinear thinwalled pipe in order to predict the time failure of the pipe according to the parameters of the stress state as well as the internal pressure of the pipe.

The results are given in Figs. 1-10. Figs. 1 and 2 show the results obtained for internal pressure versus failure time for Steel $1 \mathrm{Kh} 13 \mathrm{~N} 16 \mathrm{~B}$. For Steel $1 \mathrm{Kh} 13 \mathrm{~N} 16 \mathrm{~B}$ (Figs. 1 and 2) there is a slightly increase in failure time with $\delta=2.5$ and $\xi=0.8$ comparing with the case when $\delta=\xi=0$. While a noticeable increase in failure time (77\%) when pipes under internal pressure are stressed in combined with both axial load and bending (Fig. 2). The values of B, $\mathrm{m}, \lambda, \delta$ and $\xi$ used in the calculation are summarized in Table 1.

Figs. 3 and 4 (steel 20) incur a low failure time at high internal pressure compared with Steel $1 \mathrm{Kh} 13 \mathrm{~N} 16 \mathrm{~B}$ at the same pressure. While a Steel $\mathrm{Kh} 18 \mathrm{~N} 10 \mathrm{~T}$ incur a very low failure time at the same internal pressure (Figs. 5 and 6). This is because the material has low materials proprieties and the pipes have large parameters. The failure time increasing by $140 \%$ for St20 while it increased by $21 \%$ for Steel $\mathrm{Kh} 18 \mathrm{~N} 10 \mathrm{~T}$ at the same internal pressure, when the pipe stressed by both of axial load and bending moment.

Steel $1 \mathrm{Kh} 18 \mathrm{~N} 9 \mathrm{~T}\left(800^{\circ} \mathrm{C}\right.$ and $\left.700^{\circ} \mathrm{C}\right)$ Figs. $7-10$, incur a high failure time with the same internal pressure in compared with Figs. 1-6. The failure time for Steel $1 \mathrm{Kh} 18 \mathrm{~N} 9 \mathrm{~T}$ at $800^{\circ} \mathrm{C}$ (Fig. 8) are very low compared with Steel $1 \mathrm{Kh} 18 \mathrm{~N} 9 \mathrm{~T} 700^{\circ} \mathrm{C}$ (Fig. 10) at the same internal pressure (Steel $1 \mathrm{Kh} 18 \mathrm{~N} 9 \mathrm{~T}$ at $800^{\circ} \mathrm{C}$ introduce a very low failure time at high internal pressure, more than $2 \mathrm{MPa}$ ). The values of $\mathrm{B}, \mathrm{m}, \lambda, \delta$ and $\xi$ used in the calculation are summarized in Table 1.

It should also be noted that, as follows from the structures of the equations derived Eqs.29, 30, 32 and 33 , the time to failure increases with decrease in thickness ratio $\lambda$ and increase in the parameters $\delta$ and $\xi$. which specify the effects of the additional tensile force and bending moment.

\section{CONCLUSION}

The delayed-failure models constructed have allowed us to calculate the time to failure for thin-walled pipes under internal pressure in combination with both axial load and bending. There are good agreements between the results of the present work in calculating the failure time and the results in Golub et al. [2]. This work presents mathematical models that can be used to calculate the failure time for any rectilinear thin-walled pipes under internal pressure combined with both axial load and bending. Results for failure time of pipes which are made of different type of steels are presented and compared with the results which are calculated using the analysis of Golub et al. [2]. In this paper, the equivalent stresses are used in the form of mixed delayed-failure criterion relating the maximum normal stress and the intensity of tangential stresses and containing one material constant. The failure criterion chosen has been tested for a plane stress state with principal stresses of like sign. The present work could be applied to any material and any thin-walled tube with different dimensions which makes the analysis a basic step for computer aided creep failure.

\section{REFERENCES}

[1] A. El Megharbel, "Study of the viscoelastic beams under combined loading", Doctorial Thesis, Suez Canal University (2000).

[2] V. P. Golub, V. V. Kasperskaya and A. A. Rusinov, "Calculating the time to creep failure of thin-walled pipes under internal pressure", Int. Appl. Mech. 40, No. 4, 396-404 (2004).

[3] V. P. Golub, V. I. Krizhanovskii and A. A. Rusinov, "A mixed criterion of delayed creep failure under plane stress", int. Appl. Mech. 39, No. 5, 556-565 (2003).

[4] V. P. Golub and V. I. Krizhanovskii, "Long-term strength criteria for some polymer materials under a plane stress state", Mekh. Komp. Mater. 38 , No. 6, 471-484 (2002).

[5] V. P. Golub, V. I. Krizhanovskii, A. D. Pogrebnyak and A. V. Romanov, "A method of modeling the interaction of creep and high-cycle fatigue", Int. Appl. Mech. 41, No. 1, 14-23 (2005).

[6] V. P. Golub, "Experimental analysis of hightemperature creep, fatigue and damage. 1analysis methods", Int. Appl. Mech. 37, No. 4, 3-38 (2001).

[7]. A. Hofter, C. Broeckmann and H. Berns, H., "Creep of high speed steel part II-numerical simulation", $6^{\text {th }}$ international tooling conference, 477-494. (2002).

[8] D. Cole and H. K. D. H. Bhadeshia, "Design of Creep-Resistant Steel Welds", university of Cambridge (2001).

[9] G. Chen and X. Chen, X., "Constitutive and damage model for $63 \mathrm{Sn} 37 \mathrm{~Pb}$ solder under uniaxial and torsional cyclic loading", Int. J. Solids Struct. 43, 3596-3612 (2006). 
[10] T. Tinga, W. A. M. Brekelmans and M. G. D. Geers, "Time-incremental creep-fatigue damage rule for single crystal Ni-base superalloys", Materials Science and Engineering A 508, 200208 (2009).

[11] T. C. Yang, L. Chen, Z. C. Fan, X. D. Chen and J. L. Jiang, "A CDM-based study of fatiguecreep interaction behavior", International Journal of Pressure Vessels and Piping (under press).

[12] J. E. Bischoff, "Impact of time-dependency on long-term material testing and modeling of polyethylene", Mech. Time-Depend Mater 12, 189-203 (2008).
[13] I. Rieiro, O. A. Ruano, M Eddahbi and M. Carsi, "Integral method from initial values to obtain the best fit of the Garofalo's creep equation", J. of Materials Processing Technology 78, 177-183 (1998).

[14] L. M. Kachanov, "Rupture time under creep conditions", International Journal of Fracture 97: xi-xviii (1999).

[15] N. A. Zharkova and L. A. Botvina, "Comparative Estimation of the Life of a Material under Creep Conditions Using Various Approaches", Russian Metallurgy (Metally), No. 3, pp. 261-265 (2006).

Table 1 The used materials (Golub et al. [2])

\begin{tabular}{|c|c|c|c|c|c|c|c|}
\hline Material & ${ }^{\circ} \mathrm{C}$ & $\mathrm{B}, \mathrm{MPa}^{-\mathrm{m}} \mathrm{h}^{-1}$ & $\mathrm{~m}$ & $\lambda$ & $\delta$ & $\xi$ & $\alpha$ \\
\hline Steel Kh18N10T & 850 & $2.71 .10^{-7}$ & 3.04 & 11.50 & 5.75 & 1.5 & 0.843 \\
\hline Steel 1Kh13N16B & 700 & $1.08 .10^{-13}$ & 4.95 & 4.10 & 2.5 & 0.8 & 0.19 \\
\hline \multirow{2}{*}{ Steel 1Kh18N9T } & 800 & $3.396 .10^{-26}$ & 15.41 & 20.50 & 10.0 & 1.65 & .9126 \\
\cline { 2 - 8 } & 700 & $15.67 .10^{-16}$ & 6.23 & 7.15 & $\Gamma .5$ & 0.9 & 0.8596 \\
\hline Steel St20 & 500 & $3.117 .10^{-17}$ & 6.58 & 9.60 & 9.5 & 2.0 & 0.1619 \\
\hline
\end{tabular}

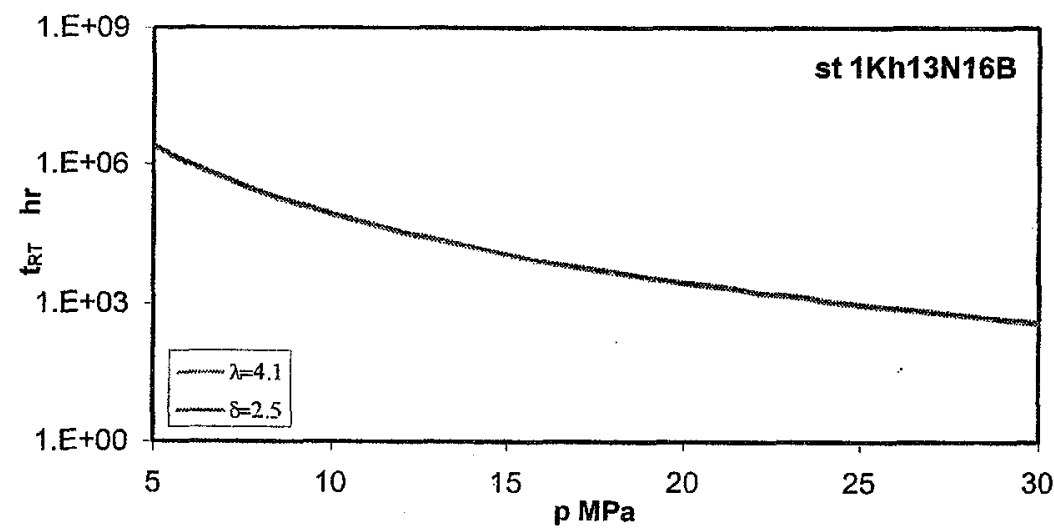

Fig. 1- The relationship between the internal pressure and the failure time for Steel $1 \mathrm{Kh} 13 \mathrm{~N} 16 \mathrm{~B}$ with $\lambda$ and $\delta$ shown in Table 1.

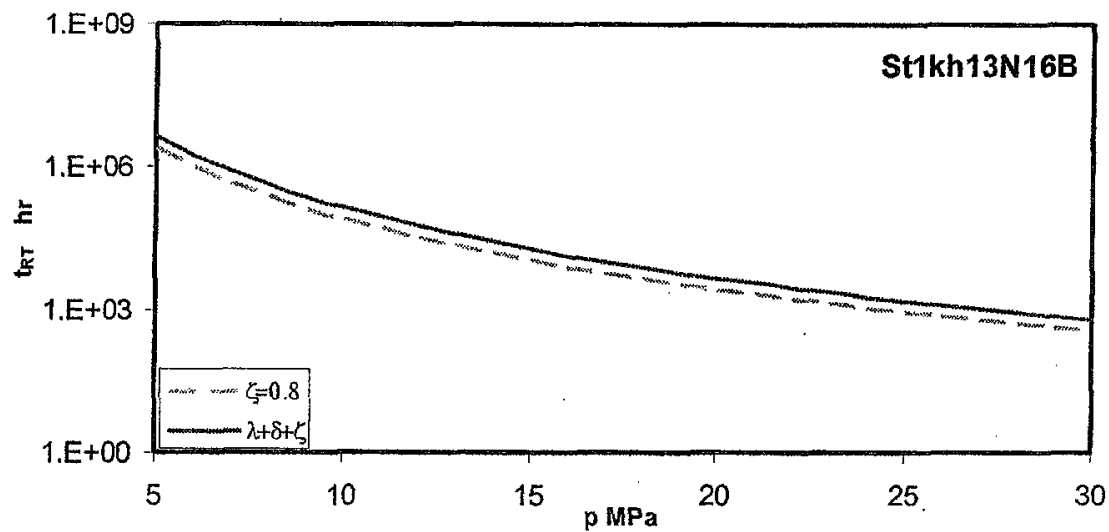

Fig. 2 The relationship between the internal pressure and the failure time for Steel $1 \mathrm{Kh} 13 \mathrm{~N} 16 \mathrm{~B}$ with $\xi$ and $\lambda, \delta, \xi$ shown in Table 1. 


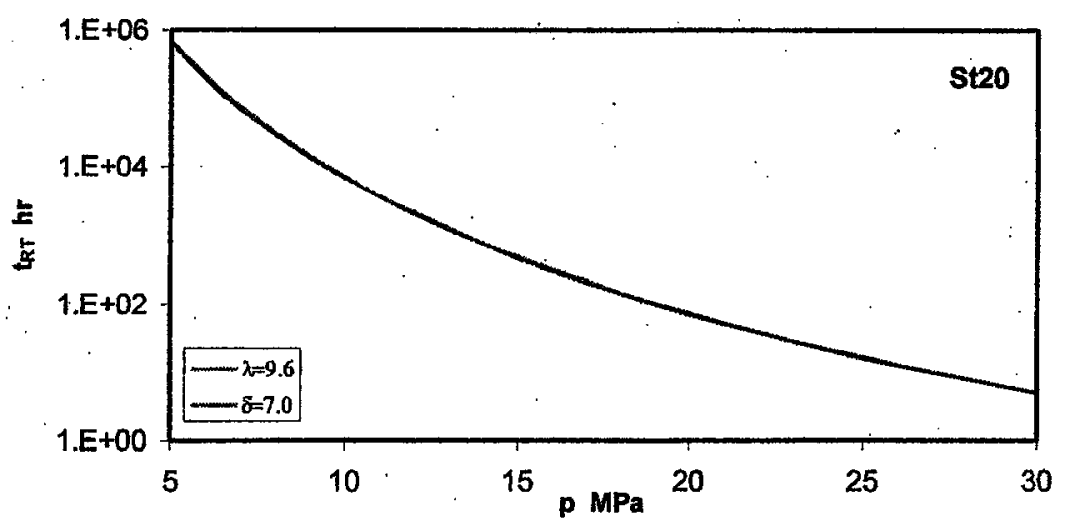

Fig. 3 The relationship between the internal pressure and the failure time for Steel St 20 with $\lambda$ and $\delta$ shown in Table 1.

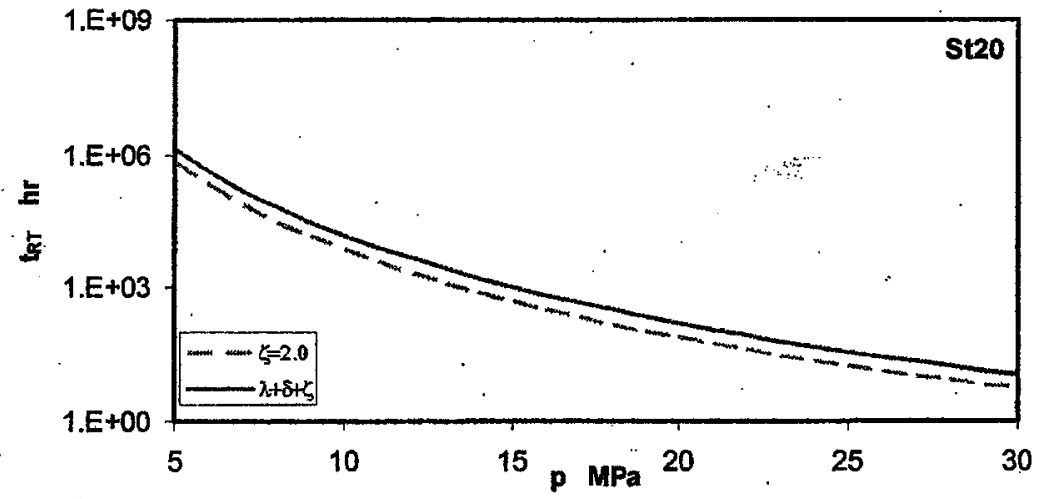

Fig. 4 The relationship between the internal pressure and the failure time for Steel St20 with $\xi$ and $\lambda, \delta$, $\xi$ shown in Table 1.

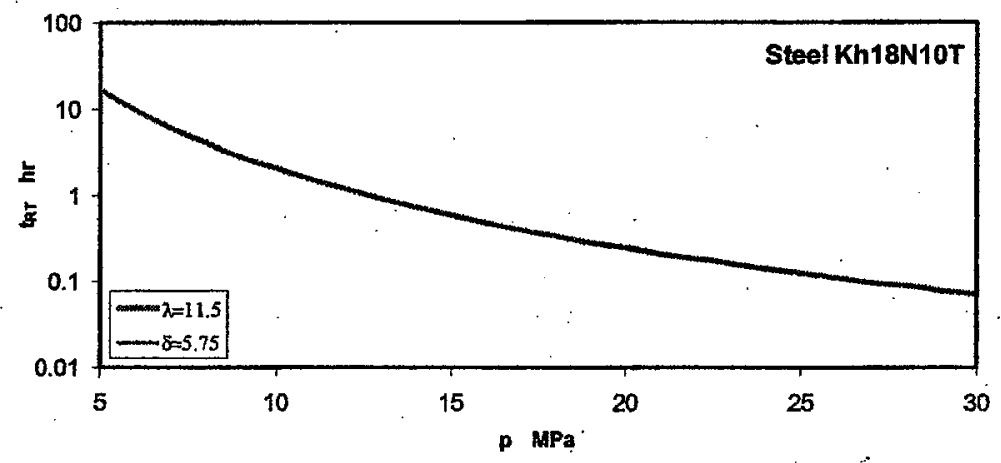

Fig. 5 The relationship between the internal pressure and the failure time for Steel Kh18N10T with $\lambda$ and $\delta$ shown in Table 1.

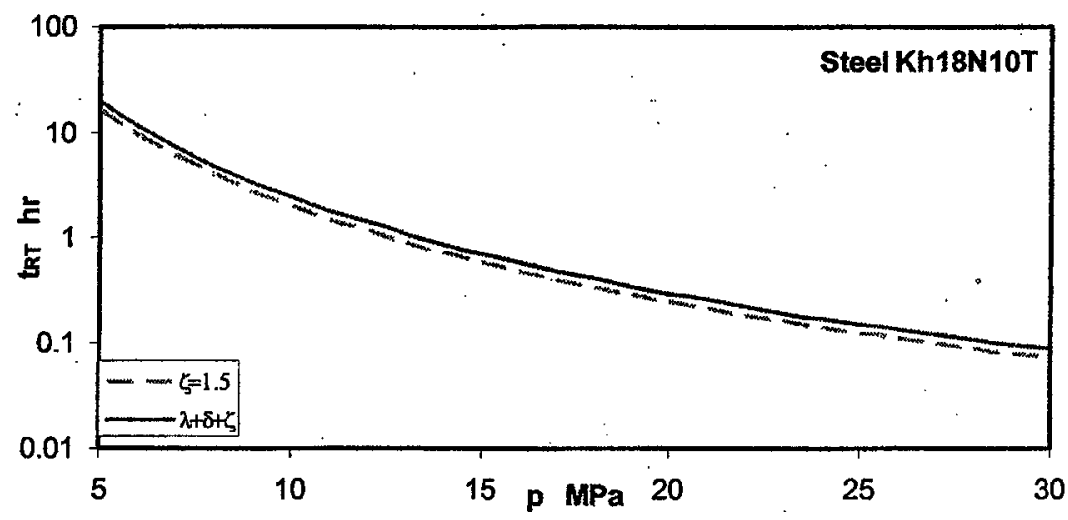

Fig. 6 The relationship between the internal pressure and the failure time for Steel Kh18N10T with $\xi$ and $\lambda, \delta, \xi$ shown in Table 1. 
A. El Megnaroel, "Lreep rature I ime of inin-wallea ripes unaer Combined internal Fressure, Bending ... "

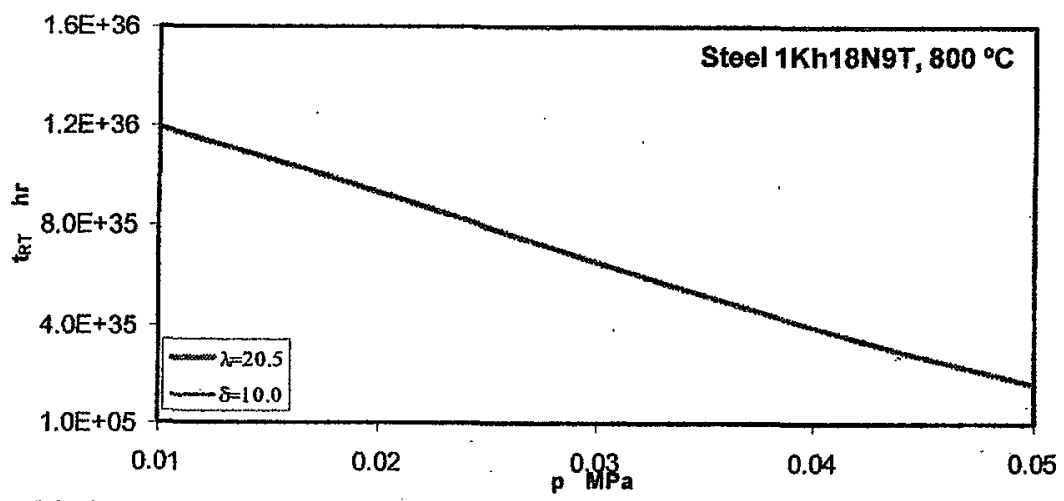

Fig. 7 The relationship between the internal pressure and the failure time for Steel $1 \mathrm{Kh} 18 \mathrm{~N} 9 \mathrm{~T}\left(800^{\circ} \mathrm{C}\right)$ with $\lambda$ and $\delta$ shown in Table 1.

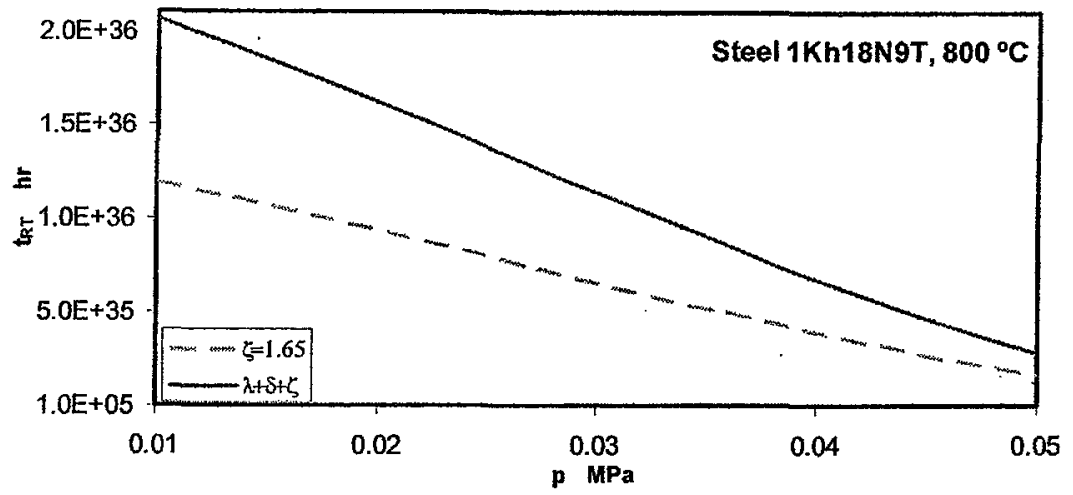

Fig. 8 The relationship between the internal pressure and the failure time for Steel $1 \mathrm{Kh} 18 \mathrm{~N} 9 \mathrm{~T}\left(800^{\circ} \mathrm{C}\right)$ with $\xi$ and $\lambda, \delta, \xi$ shown in Table 1 .

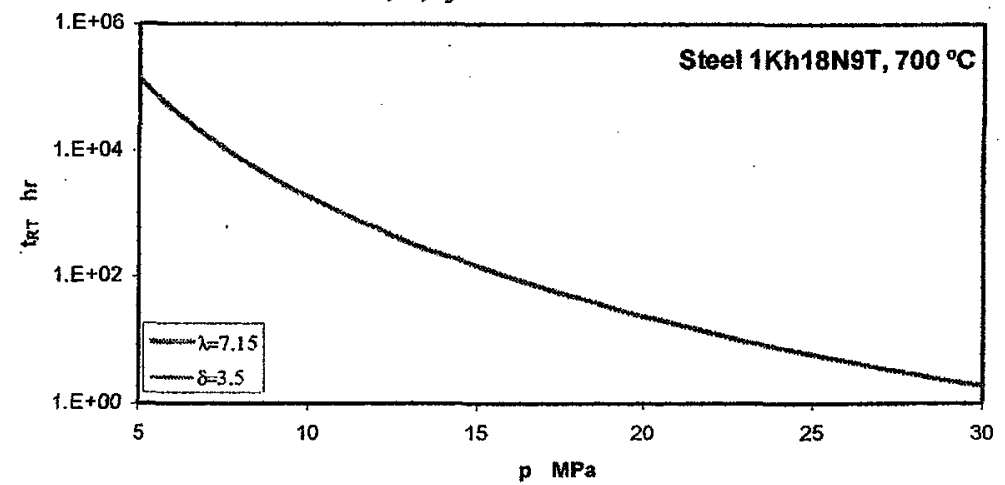

Fig. 9 The relationship between the internal pressure and the failure time for Steel $1 \mathrm{Kh} 18 \mathrm{~N} 9 \mathrm{~T}\left(700^{\circ} \mathrm{C}\right)$ with $\lambda$ and $\delta$ shown in Table 1.

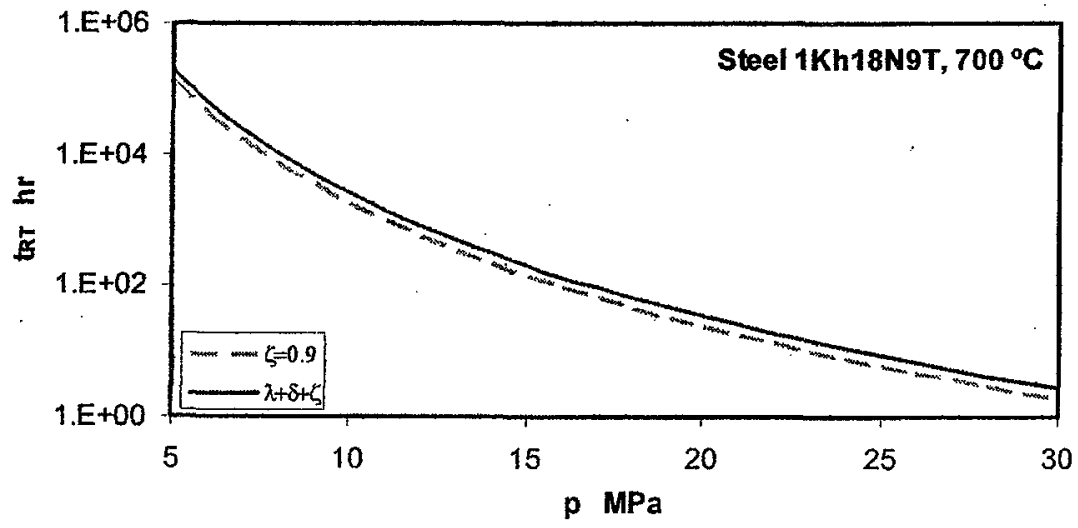

Fig. 10 The relationship between the internal pressure and the failure time for Steel $1 \mathrm{Kh} 18 \mathrm{~N} 9 \mathrm{~T}\left(700^{\circ} \mathrm{C}\right)$ with $\xi$ and $\lambda, \delta, \xi$ shown in Table 1 . 